\title{
Asymptotics of nearly critical Galton-Watson processes with immigration
}

\author{
PÉTER Kevei ${ }^{1}$ \\ Centro de Investigación en Matemáticas, Jalisco S/N, Valenciana, \\ Guanajuato, GTO 36240, Mexico; e-mail: kevei@cimat.mx
}

\begin{abstract}
We investigate the inhomogeneous Galton-Watson processes with immigration, where $\rho_{n}$ the offspring means in the $n^{\text {th }}$ generation tends to 1 . We show that if the second derivatives of the offspring generating functions go to 0 rapidly enough, then the asymptotics are the same as in the INAR(1) case, treated in [4]. We also determine the limit if this assumption does not hold showing the optimality of the conditions. AMS Subject Classification (2000): 60J80.

Keywords: nearly critical Galton-Watson process; immigration; compound Poisson distribution; negative binomial distribution.
\end{abstract}

\section{Introduction}

Let $X_{0}=0$ and consider the following inhomogeneous Galton-Watson process with immigration:

$$
X_{n}=\sum_{j=1}^{X_{n-1}} \xi_{n, j}+\varepsilon_{n},
$$

where $\left\{\xi_{n, j}, \varepsilon_{n}: n, j \in \mathbb{N}\right\}$ are independent nonnegative integer valued random variables such that $\left\{\xi_{n, j}: j \in \mathbb{N}\right\}$ are identically distributed. If the offspring distribution is Bernoulli distribution, that is each particle either

\footnotetext{
${ }^{1}$ The research was supported by the Analysis and Stochastics Research Group of The Hungarian Academy of Sciences.
} 
dies without descendant or leaves exactly one descendant, we obtain the socalled first order integer-valued autoregressive (INAR(1)) time series. We assume that the process is nearly critical, that is

$$
\mathbf{E} \xi_{n, 1}=\rho_{n} \uparrow 1 \quad \text { as } n \rightarrow \infty .
$$

We note here that in the followings any non-specified limit relation is meant as $n \rightarrow \infty$.

The theory of branching processes is used to model the evolution of populations whose members live, reproduce and die independently of each other. Its first appearance was motivated by the problem of extinction probability of the family names in the British peerage. The problem was addressed by Francis Galton in 1873 and solved by Henry Watson in 1874. Since then the theory is developing to model more complex systems, and now branching processes play an important role in models of genetics, molecular biology, physics and computer science. Therefore we do not even try to give a comprehensive bibliography. As a main reference on branching processes we refer to the classical book of Athreya and Ney [1]. For some recent application of INAR models and branching processes see [4].

The aim of the present paper is to investigate the asymptotic properties of nearly critical Galton-Watson processes with immigration under general offspring distribution, and thus extend the results in the INAR(1) case by Györfi, Ispány, Pap and Varga [4], by dropping the restrictive condition of Bernoulli offsprings.

Section 2 contains all the results and the discussions. In subsection 2.1 we investigate the case which is parallel to the results in [4]. Here we assume that the variance of the offsprings tends to 0 with a prescribed rate, and thus in a clear sense the offsprings are 'almost' Bernoulli random variables. The methods here are similar the ones in [4], however we emphasize that the proofs are necessarily more difficult, since we do not have a closed form for the generating functions even in the case of Bernoulli immigration. Subsection 2.2 deals with a significantly different case, when the second derivatives also contribute to the limit distribution, and so the proofs need new ideas. Subsection 2.3 contains the case, when $\rho_{n} \rightarrow 1$ very fast. Finally, in subsection 2.4 we consider the case of linear fractional generating functions. This example is very important because of the explicit computations. All the proofs are placed in section 3 . 


\section{Results and discussion}

Introduce the generating functions

$$
F_{n}(x)=\mathbf{E} x^{X_{n}}, \quad G_{n}(x)=\mathbf{E} x^{\xi_{n, 1}}, \quad H_{n}(x)=\mathbf{E} x^{\varepsilon_{n}}, x \in[0,1] .
$$

Using the branching property we obtain the basic recursion for $F_{n}$ :

$$
\begin{aligned}
F_{n}(x) & =\mathbf{E}\left[x^{\sum_{i=1}^{X_{n-1}} \xi_{n, i}+\varepsilon_{n}}\right]=\mathbf{E}\left[\mathbf{E}\left(x^{\sum_{i=1}^{X_{n-1}} \xi_{n, i}+\varepsilon_{n}} \mid X_{n-1}\right)\right] \\
& =\mathbf{E}\left[G_{n}(x)^{X_{n-1}}\right] H_{n}(x)=F_{n-1}\left(G_{n}(x)\right) H_{n}(x) .
\end{aligned}
$$

Introduce the following notation: $\bar{G}_{n+1, n}(x)=x$ and if $\bar{G}_{j+1, n}(x)$ is defined for $j \leq n$ then

$$
\bar{G}_{j, n}(x)=\left(G_{j} \circ \ldots \circ G_{n}\right)(x)=G_{j}\left(\bar{G}_{j+1, n}(x)\right) .
$$

With this notation the induction above gives the formula

$$
F_{n}(x)=\prod_{j=1}^{n} H_{j}\left(\bar{G}_{j+1, n}(x)\right)
$$

According to the continuity theorem for discrete random variables ([2] p. 280) for proving a limit theorem we have to show that $F_{n}(x)$ converges as $n \rightarrow \infty$ for all $x \in(0,1)$, and the limit function is the generating function

of the limit distribution. Since for fix $j$ the function $H_{j}\left(\bar{G}_{j+1, n}(x)\right) \sim 1$, we introduce the corresponding generating function

$$
\tilde{F}_{n}(x)=\prod_{j=1}^{n} \mathrm{e}^{H_{j}\left(\bar{G}_{j+1, n}(x)\right)-1}=\exp \left\{\sum_{j=1}^{n}\left(H_{j}\left(\bar{G}_{j+1, n}(x)\right)-1\right)\right\},
$$

which is easier to handle, because of its exponential form. This is a kind of accompanying law of $X_{n}$. So for proving a limit theorem we have to check the following two conditions:

(a) $F_{n}(x)-\tilde{F}_{n}(x) \rightarrow 0$ for all $x \in(0,1)$, and

(b) the $\operatorname{limit}_{n \rightarrow \infty} \tilde{F}_{n}(x)$ exists for all $x \in(0,1)$, and the limit function is a generating function. 
Also note the intuitively clear fact, that

$$
\bar{G}_{j+1, n}^{\prime}(1)=\rho_{j+1} \rho_{j+2} \cdots \rho_{n}=\rho_{[j, n]} .
$$

This is because $\bar{G}_{n, n}^{\prime}(1)=G_{n}^{\prime}(1)=\rho_{n}$ and

$$
\bar{G}_{j, n}^{\prime}(1)=G_{j}^{\prime}(1) \bar{G}_{j+1, n}^{\prime}(1)=\rho_{j} \bar{G}_{j+1, n}^{\prime}(1),
$$

and so by induction we have (2).

\subsection{Poisson and compound Poisson limits}

In this subsection we investigate the case, when the second derivative of the offspring generating function goes to 0 so fast that it does not appear in the limit distribution. So the results here are the analogs of the ones in the case of Bernoulli offsprings in [4].

In the followings Poisson $(\lambda)$ stands for a Poisson distribution with parameter $\lambda$ if $\lambda>0$, and Poisson(0) is the degenerate distribution at 0 , while $\operatorname{Bernoulli}(p)$ is a Bernoulli distribution with parameter $p>0$.

The first theorem deals with the case when the immigration has Bernoulli distribution.

Theorem 1 Let $\left\{X_{n}\right\}_{n \in \mathbb{N}}$ be a Galton-Watson process with immigration, with $\varepsilon_{n} \sim \operatorname{Bernoulli}\left(m_{n, 1}\right)$. Assume that

(i) $\rho_{n}<1, \lim _{n \rightarrow \infty} \rho_{n}=1, \sum_{n=1}^{\infty}\left(1-\rho_{n}\right)=\infty$ and $\lim _{n \rightarrow \infty} \frac{G_{n}^{\prime \prime}(1)}{1-\rho_{n}}=0$,

(ii) $\lim _{n \rightarrow \infty} \frac{m_{n, 1}}{1-\rho_{n}}=\lambda$.

Then

$$
X_{n} \stackrel{\mathcal{D}}{\longrightarrow} \operatorname{Poisson}(\lambda)
$$

Next we turn to general immigration distributions. Let us denote the factorial moments of the immigration distribution by

$$
m_{n, k}:=\mathbf{E}\left[\varepsilon_{n}\left(\varepsilon_{n}-1\right) \ldots\left(\varepsilon_{n}-k+1\right)\right]=H_{n}^{(k)}(1) .
$$

(Clearly, this is consistent with the notation Bernoulli $\left(m_{n, 1}\right)$.)

The analog of Theorem 2 [4] can be shown: the process has Poisson limit even if the immigration distribution is not Bernoulli, only 'close' to it. Since the proof is also the same as there, we omit it. 
Theorem 2 Let $\left\{X_{n}\right\}_{n \in \mathbb{N}}$ be a Galton-Watson process with immigration. Assume that

(i) $\rho_{n}<1, \lim _{n \rightarrow \infty} \rho_{n}=1, \sum_{n=1}^{\infty}\left(1-\rho_{n}\right)=\infty$ and $\lim _{n \rightarrow \infty} \frac{G_{n}^{\prime \prime}(1)}{1-\rho_{n}}=0$,

(ii) $\lim _{n \rightarrow \infty} \frac{m_{n, 1}}{1-\rho_{n}}=\lambda, \lim _{n \rightarrow \infty} \frac{m_{n, 2}}{1-\rho_{n}}=0$.

Then

$$
X_{n} \stackrel{\mathcal{D}}{\longrightarrow} \operatorname{Poisson}(\lambda) .
$$

Before the following results we recall that for a finite measure $\mu$ on $\mathbb{Z}_{+}=$ $\{1,2, \ldots\}$ the compound Poisson distribution $\mathrm{CP}(\mu)$ with intensity measure $\mu$ is the distribution which has generating function

$$
\exp \left\{\sum_{j=1}^{\infty} \mu\{j\}\left(x^{j}-1\right)\right\}, \quad x \in[0,1] .
$$

The analogs of Theorem 4 and 5 in [4] are also true. The proof of Theorem 3 is the same as of Theorem 4 in [4], and also follows from the stronger Theorem 4, so we skip it.

Theorem 3 Let $\left\{X_{n}\right\}_{n \in \mathbb{N}}$ be a Galton-Watson process with immigration. Assume that

(i) $\rho_{n}<1, \lim _{n \rightarrow \infty} \rho_{n}=1, \sum_{n=1}^{\infty}\left(1-\rho_{n}\right)=\infty$ and $\lim _{n \rightarrow \infty} \frac{G_{n}^{\prime \prime}(1)}{1-\rho_{n}}=0$,

(ii) $\lim _{n \rightarrow \infty} \frac{m_{n, j}}{j\left(1-\rho_{n}\right)}=\lambda_{j}$ for $j=1,2, \ldots, J$ with $\lambda_{J}=0$.

Then

$$
X_{n} \stackrel{\mathcal{D}}{\longrightarrow} \mathrm{CP}(\mu),
$$

where $\mu$ is a finite measure on $\{1,2, \ldots, J-1\}$ given by

$$
\mu\{j\}=\frac{1}{j !} \sum_{i=0}^{J-j-1} \frac{(-1)^{i}}{i !} \lambda_{j+i}, \quad j=1,2, \ldots, J-1 .
$$

The following theorem is more general than Theorem 5 in [4] even in the case of Bernoulli offspring distributions. And since the proof of Theorem 5 in [4] is only given by Poisson approximation we give the analytical proof. 
Theorem 4 Let $\left\{X_{n}\right\}_{n \in \mathbb{N}}$ be a Galton-Watson process with immigration. Assume that

(i) $\rho_{n}<1, \lim _{n \rightarrow \infty} \rho_{n}=1, \sum_{n=1}^{\infty}\left(1-\rho_{n}\right)=\infty$ and $\lim _{n \rightarrow \infty} \frac{G_{n}^{\prime \prime}(1)}{1-\rho_{n}}=0$,

(ii) $\lim _{n \rightarrow \infty} \frac{m_{n, j}}{j\left(1-\rho_{n}\right)}=\lambda_{j}$ for $j=1,2, \ldots$, and $\lim \sup _{n \rightarrow \infty} \sqrt[n]{\lambda_{n} / n !} \leq 1$.

Then

$$
X_{n} \stackrel{\mathcal{D}}{\longrightarrow} Y,
$$

where the random variable $Y$ has generating function

$$
\mathbf{E} x^{Y}=\exp \left\{\sum_{l=1}^{\infty} \frac{(x-1)^{l}}{l !} \lambda_{l}\right\} .
$$

Note that if $\lambda_{n}=0$ for some $n \geq 2$, then $\lambda_{m}=0$ for all $m \geq n$. This simple fact follows from the previous two theorems.

Also notice that the assumption $\lim \sup _{n \rightarrow \infty} \sqrt[n]{\lambda_{n} / n !} \leq 1$ implies that the limiting generating function exists for $x \in(0,1)$. This assumption is weaker than the one in [4], which is that for all $j=1,2, \ldots$ the limit

$$
\mu\{j\}=\frac{1}{j !} \sum_{i=0}^{\infty} \frac{(-1)^{i}}{i !} \lambda_{j+i}
$$

exists. However, under this assumption the limit turns out to be a compound Poisson random variable with intensity measure $\mu$, that is

$$
\exp \left\{\sum_{l=1}^{\infty} \frac{(x-1)^{l}}{l !} \lambda_{l}\right\}=\exp \left\{\sum_{l=1}^{\infty} \mu\{l\}\left(x^{l}-1\right)\right\} .
$$

This follows easily by Abel's theorem.

The limit can be compound Poisson even if condition (4) fails. If $\lambda_{n}=$ $(n-1) ! / n$, then condition (4) does not hold even for $j=2$, but

$\sum_{l=1}^{\infty} \frac{(x-1)^{l}}{l^{2}}=-\int_{0}^{x-1} \frac{\log (1-u)}{u} \mathrm{~d} u=\int_{1}^{x} \frac{\log (2-y)}{1-y} \mathrm{~d} y=\sum_{j=1}^{\infty} \mu\{j\}\left(x^{j}-1\right)$,

with

$$
\mu\{j\}=\frac{1}{j}\left[\log 2-\sum_{k=1}^{j-1} \frac{1}{k 2^{k}}\right]
$$


that is the limit is compound Poisson with intensity measure $\mu$. To see that the sequence $\left\{\lambda_{n}=\frac{(n-1) !}{n}\right\}_{n=1}^{\infty}$ can be a limit in condition (ii) consider the following example: $\rho_{n}=1-n^{-1}$ and $H_{n}(x)=1+(H(x)-1) / n$ with the generating function $H(x)=1-\log (2-x)$.

We do not know whether the limit in the previous theorem necessarily has a compound Poisson distribution.

\subsection{Negative binomial limits}

In the followings we investigate the case, when $G_{n}^{\prime \prime}(1) /\left(1-\rho_{n}\right) \not \rightarrow 0$. In contrast to the previous subsection we show that in this case the second derivatives do appear in the limit. Here we restrict ourselves to the case when the immigration distribution is close to a Bernoulli distribution.

Note that for condition (a) it is only needed that $\rho_{n}<1, \rho_{n} \rightarrow 1$ and $\sum_{n=1}^{\infty}\left(1-\rho_{n}\right)=\infty$ (see the proof of Theorem 1$)$. Since we always assume these conditions we concentrate on condition (b), i.e. -in case of Bernoulli immigration- on the existence of the limit

$$
\lim _{n \rightarrow \infty} \sum_{j=1}^{n} m_{j, 1}\left(\bar{G}_{j+1, n}(x)-1\right) .
$$

We try to compute the derivatives at 1 of the components in the sum above. First note that since $\bar{G}_{j+1, n}^{\prime}(1)=\rho_{[j, n]}$, the first derivative in (5) is

$$
\sum_{j=1}^{n} m_{j, 1} \rho_{[j, n]}=\sum_{j=1}^{n} \frac{m_{j, 1}}{1-\rho_{j}}\left(1-\rho_{j}\right) \rho_{[j, n]},
$$

and since $\left\{\left(1-\rho_{j}\right) \rho_{[j, n]}\right\}$ form a Toeplitz matrix, we obtain that in order to get a limit the asymptotic order of $m_{n, 1}$ must be $1-\rho_{n}$. For the second derivative we have the recursion

$$
\begin{aligned}
\bar{G}_{j, n}^{\prime \prime}(x) & =\frac{\mathrm{d}^{2}}{\mathrm{~d} x^{2}} G_{j}\left(\bar{G}_{j+1, n}(x)\right)=\frac{\mathrm{d}}{\mathrm{d} x}\left(G_{j}^{\prime}\left(\bar{G}_{j+1, n}(x)\right) \bar{G}_{j+1, n}^{\prime}(x)\right) \\
& =G_{j}^{\prime \prime}\left(\bar{G}_{j+1, n}(x)\right) \bar{G}_{j+1, n}^{\prime}(x)^{2}+G_{j}^{\prime}\left(\bar{G}_{j+1, n}(x)\right) \bar{G}_{j+1, n}^{\prime \prime}(x) .
\end{aligned}
$$

Substituting $x=1$ for $j \leq n$ (recall that $\bar{G}_{n+1, n}(x)=x$ ) by (2) we obtain that

$$
\bar{G}_{j, n}^{\prime \prime}(1)=G_{j}^{\prime \prime}(1) \rho_{[j, n]}^{2}+\rho_{j} \bar{G}_{j+1, n}^{\prime \prime}(1),
$$


and $\bar{G}_{n, n}^{\prime \prime}(1)=G_{n}^{\prime \prime}(1)$. Induction argument shows that

$$
\bar{G}_{j+1, n}^{\prime \prime}(1)=\sum_{i=j+1}^{n} G_{i}^{\prime \prime}(1) \rho_{[j, i-1]} \rho_{[i, n]}^{2},
$$

so the second derivative in $(5)$ is

$$
\sum_{j=1}^{n} m_{j, 1} \bar{G}_{j+1, n}^{\prime \prime}(1)=\sum_{i=2}^{n} G_{i}^{\prime \prime}(1) \rho_{[i, n]}^{2} \sum_{j=1}^{i-1} m_{j, 1} \rho_{[j, i-1]} .
$$

As we have seen $m_{n} \approx 1-\rho_{n}$, and so to get a limit for the second derivative we must have $G_{n}^{\prime \prime}(1) \approx 1-\rho_{n}$. These heuristic argument kind of shows the necessity of the assumptions in the following theorem. We also note that Step 2 of the proof Theorem 5 shows that the higher derivatives must have the same order, namely $1-\rho_{n}$. We are not able to calculate these contributions, therefore we have to assume condition (iii).

The reasoning above can be made rigorous to show the following: If $\lim _{n \rightarrow \infty} G_{n}^{\prime \prime}(1)=a$ exists and the inhomogeneous Galton-Watson process with immigration has a proper limit distribution with finite second moment, then necessarily $a=0$. This immediately implies that $\lim _{n \rightarrow \infty} G_{n}(x)=x$, i.e. there is no critical nontrivial branching mechanism with finite second moment, which can cause a proper limit distribution with finite second moment. This result is in complete accordance with Theorem 1 (ii) by Foster and Williamson [3] in the homogeneous case.

We will show that in this setup the limit distribution is the negative binomial distribution. A random variable $X$ has negative binomial distribution with parameters $r>0$ and $p \in(0,1)$, denoted by $\mathrm{NB}(r, p)$, if $\mathbf{P}\{X=k\}=\left(\begin{array}{c}k+r-1 \\ r-1\end{array}\right)(1-p)^{r} p^{k}, k=0,1,2, \ldots$, where the binomial coefficient is defined by $\left(\begin{array}{c}k+r-1 \\ r-1\end{array}\right)=\frac{(k+r-1)(k+r-2) \cdots r}{k !}$. The generating function is

$$
\mathbf{E} x^{X}=\left(\frac{1-p}{1-p x}\right)^{r} \text {. }
$$

The following theorem holds.

Theorem 5 Let $\left\{X_{n}\right\}$ be a Galton-Watson process with immigration, with general offspring and immigration distribution, such that the followings hold:

(i) $\rho_{n}<1, \lim _{n \rightarrow \infty} \rho_{n}=1, \sum_{n=1}^{\infty}\left(1-\rho_{n}\right)=\infty$, 
(ii) $\lim _{n \rightarrow \infty} \frac{G_{n}^{\prime \prime}(1)}{1-\rho_{n}}=\nu \in(0, \infty)$,

(iii) $\lim _{n \rightarrow \infty} \frac{G_{n}^{(s)}(1)}{1-\rho_{n}}=0$, for all $s \geq 3$,

(iv) $\lim _{n \rightarrow \infty} \frac{m_{n, 1}}{1-\rho_{n}}=\lambda$ and $\lim _{n \rightarrow \infty} \frac{m_{n, 2}}{1-\rho_{n}}=0$.

Then

$$
X_{n} \stackrel{\mathcal{D}}{\longrightarrow} \mathrm{NB}(2 \lambda / \nu, \nu /(2+\nu))
$$

As we already mentioned, assumption (ii) means that the second derivatives have a significant role in the limit, while assumption (iii) ensures that the third and higher derivatives do not count. The proof of the theorem basically lays on determining the asymptotic of $\bar{G}_{j+1, n}^{(k)}$, combined with a relatively closed formula for the coefficient of $f^{\prime \prime}(g)$ in $\frac{\mathrm{d}^{k}}{\mathrm{~d} x^{k}} f(g(x))$, see (13). This 'shows' that to handle the case, when the higher derivatives also count, a different approach is needed, or at least the calculations become more technical. It would be also interesting to extend the results to more general immigration distribution, that is to know whether a kind of analog of Theorem 4 remains true.

Finally, we note that if $\lambda>0$ is fixed and $\nu \rightarrow 0$, then

$$
\lim _{\nu \rightarrow 0} \operatorname{NB}(2 \lambda / \nu, \nu /(2+\nu)) \rightarrow \text { Poisson }(\lambda) .
$$

It is easy to check that the proof of the theorem remains correct in this case, and we obtain that Theorem 5 holds if $\nu=0$ and in this case the limit distribution is Poisson $(\lambda)$, which is exactly the statement of Theorem 2.

\subsection{The case $\sum_{n=1}^{\infty}\left(1-\rho_{n}\right)<\infty$}

Let us consider the nearly critical inhomogeneous Galton-Watson process with general offspring distribution and general immigration. It was always assumed that $\rho_{n}$ does not converge too fast to 1 , that is $\sum_{n=1}^{\infty}\left(1-\rho_{n}\right)=\infty$. In this section we investigate the case, when this assumption does not hold, and assume that

$$
\prod_{n=2}^{\infty} \rho_{n}=\rho \in(0,1) .
$$


(Note that this is equivalent to the assumption $\sum_{n=1}^{\infty}\left(1-\rho_{n}\right)<\infty$.) We show that in this case the limit distribution exists under very general assumptions, but the process does not contain enough randomness as in the previous cases: the limit distribution explicitly contains each offspring- and immigration distribution. That is $\rho_{n}$ tends to 1 so fast, that the process has no time to forget the initial distribution. This shows that the right assumption is indeed $\sum_{n=1}^{\infty}\left(1-\rho_{n}\right)=\infty$, as it was investigated in [4]. In fact, the case treated in this section is much simpler.

We state the theorem in the most general setup.

Theorem 6 Let $X_{n}$ be the Galton-Watson process with immigration, described above. If $\prod_{n=2}^{\infty} \rho_{n}=\rho \in(0,1)$ and $\sum_{n=1}^{\infty} m_{n, 1}<\infty$, then

$$
X_{n} \stackrel{\mathcal{D}}{\longrightarrow} Y,
$$

where $Y$ has generating function

$$
g(x)=\prod_{j=1}^{\infty} H_{j}\left(\bar{G}_{j+1, \infty}(x)\right),
$$

with

$$
\bar{G}_{j+1, \infty}(x)=\lim _{n \rightarrow \infty} \bar{G}_{j+1, n}(x) .
$$

Let us see an example in the simplest case, when both the offspring distribution and the immigration distribution is Bernoulli. In this case we can compute the limit generating function.

Example 1. Let $\rho_{n}=1-\frac{1}{n^{2}}$. Clearly $\sum_{n=1}^{\infty}\left(1-\rho_{n}\right)=\pi^{2} / 6<\infty$. For the product we have

$$
\begin{aligned}
\rho_{[1, n]} & =\rho_{2} \rho_{3} \ldots \rho_{n}=\left(1-\frac{1}{2^{2}}\right)\left(1-\frac{1}{3^{2}}\right) \cdots\left(1-\frac{1}{n^{2}}\right) \\
& =\frac{\left(2^{2}-1\right)\left(3^{2}-1\right) \ldots\left(n^{2}-1\right)}{(n !)^{2}} \\
& =\frac{1 \cdot 3 \cdot 2 \cdot 4 \cdot \ldots \cdot(n-1) \cdot(n+1)}{(n !)^{2}} \\
& =\frac{n+1}{2 n} \rightarrow \frac{1}{2}=\rho .
\end{aligned}
$$


In this case $\bar{G}_{j+1, n}(x)=1+\rho_{[j, n]}(x-1)$, so $\bar{G}_{j+1, \infty}(x)=1+\rho(x-1) / \rho_{[1, j]}$. Since $H_{j}(x)=1-m_{j, 1}(1-x)$, for the limit generating function we have

$$
g(x)=\prod_{j=1}^{\infty}\left[1-\frac{m_{j, 1}}{\rho_{[1, j]}} \frac{1}{2}(1-x)\right]=\prod_{j=1}^{\infty}\left[1-m_{j, 1} \frac{j}{j+1}(1-x)\right]
$$

Choose $m_{j, 1}=(j+1) / j^{3}$, so

$$
g(x)=\prod_{j=1}^{\infty}\left[1-\frac{1-x}{j^{2}}\right]=\frac{\sin (\pi \sqrt{1-x})}{\pi \sqrt{1-x}} .
$$

The following example is Example 1 in [4], which shows that in a special case the limit can be Poisson even in this setup.

Example 2. Assume that the offspring distribution is $\operatorname{Bernoulli}\left(\rho_{n}\right)$ and the immigration $\varepsilon_{n}$ has $\operatorname{Poisson}\left(m_{n, 1}\right)$ distribution, where $\sum_{n=1}^{\infty}\left(1-\rho_{n}\right)<\infty$ and $\sum_{n=1}^{\infty} m_{n, 1}<\infty$. As before $\bar{G}_{j+1, \infty}(x)=1+\rho(x-1) / \rho_{[1, j]}$, and since $H_{n}(x)=\mathrm{e}^{m_{n, 1}(x-1)}$ we have that

$$
g(x)=\exp \left\{(x-1) \sum_{n=1}^{\infty} \frac{m_{n, 1} \rho}{\rho_{[1, n]}}\right\}
$$

and since the sum in the exponent is finite, this is Poisson distribution with mean $\sum_{n=1}^{\infty} \frac{m_{n, 1} \rho}{\rho_{[1, n]}}$.

\subsection{The linear fractional case}

The importance of the linear fractional generating functions in branching processes is that, that this is basically the only example when explicit computation can be done. In this subsection we investigate this example in detail, which actually helped to find the general form of Theorem 5 .

The linear fractional generating function has the form $f(s)=1-\frac{\alpha}{1-\beta}+$ $\frac{\alpha s}{1-\beta s}$, where $\alpha, \beta \in(0,1), \alpha+\beta \leq 1$. For the first two derivatives we have

$$
\begin{aligned}
f^{\prime}(1) & =\frac{\alpha}{(1-\beta)^{2}} \\
f^{\prime \prime}(1) & =\frac{2 \alpha \beta}{(1-\beta)^{3}}
\end{aligned}
$$


and these determine the parameters $\alpha, \beta$ :

$$
\begin{aligned}
\alpha & =\frac{4 f^{\prime}(1)^{3}}{\left(2 f^{\prime}(1)+f^{\prime \prime}(1)\right)^{2}} \\
\beta & =\frac{f^{\prime \prime}(1)}{2 f^{\prime}(1)+f^{\prime \prime}(1)} .
\end{aligned}
$$

If $f_{1}(s)$ and $f_{2}(s)$ are both linear fractional generating functions then so is $f_{1}\left(f_{2}(s)\right)$, that is if $G_{n}$ is linear fractional for all $n$, so is $\bar{G}_{j, n}(s)$ for all $j, n$, with parameters

$$
\begin{aligned}
\alpha_{j, n} & =\frac{4 \bar{G}_{j, n}^{\prime}(1)^{3}}{\left(2 \bar{G}_{j, n}^{\prime}(1)+\bar{G}_{j, n}^{\prime \prime}(1)\right)^{2}}, \\
\beta_{j, n} & =\frac{\bar{G}_{j, n}^{\prime \prime}(1)}{2 \bar{G}_{j, n}^{\prime}(1)+\bar{G}_{j, n}^{\prime \prime}(1)} .
\end{aligned}
$$

As we have seen $\bar{G}_{j, n}^{\prime}(1)=\rho_{[j-1, n]}$ and $\bar{G}_{j, n}^{\prime \prime}(1)=\sum_{i=j}^{n} G_{i}^{\prime \prime}(1) \rho_{[j-1, i-1]} \rho_{[i, n]}^{2}$. The generating function has the form $F_{n}(x)=\prod_{j=1}^{n} H_{j}\left(\bar{G}_{j+1, n}(x)\right)$. Assuming Bernoulli immigration, i.e. $H_{n}(x)=1+m_{n}(x-1)$, the corresponding accompanying generating function is

$$
\begin{aligned}
\tilde{F}_{n}(x) & =\exp \left\{\sum_{j=1}^{n} m_{j}\left(\bar{G}_{j+1, n}(x)-1\right)\right\} \\
& =\exp \left\{\sum_{j=1}^{n} m_{j}\left(-\frac{\alpha_{j+1, n}}{1-\beta_{j+1, n}}+\frac{\alpha_{j+1, n} x}{1-\beta_{j+1, n} x}\right)\right\} .
\end{aligned}
$$

Some calculation shows

$$
\frac{\alpha_{j+1, n}}{1-\beta_{j+1, n} x}=\frac{\rho_{[j, n]}}{\left(1+\sum_{i=j+1}^{n} \frac{G_{i}^{\prime \prime}(1) \rho_{[i, n]}}{2 \rho_{i}}\right)\left(1+\sum_{i=j+1}^{n} \frac{G_{i}^{\prime \prime}(1) \rho_{[i, n]}}{2 \rho_{i}}(1-x)\right)} .
$$

Eventually, we obtained that in the linear fractional case the limit exists (under the assumption $\sum_{n=1}^{\infty}\left(1-\rho_{n}\right)=\infty$ ) if and only if

$$
\lim _{n \rightarrow \infty} \sum_{j=1}^{n} m_{j} \rho_{[j, n]}\left[\left(1+\sum_{i=j+1}^{n} \frac{G_{i}^{\prime \prime}(1) \rho_{[i, n]}}{2 \rho_{i}}\right)\left(1+\sum_{i=j+1}^{n} \frac{G_{i}^{\prime \prime}(1) \rho_{[i, n]}}{2 \rho_{i}}(1-x)\right)\right]^{-1}
$$

exists. It is easy to check that if (ii) in Theorem 5 holds, then so is (iii), that is the limit is negative binomial distribution. 


\section{Proofs}

We use the continuity theorem for generating functions ([2] p. 280) and Lemmas 5 and 6 in [4] without any further reference. We also frequently use the simple facts that

$$
\left|\prod_{k=1}^{n} z_{k}-\prod_{k=1}^{n} w_{k}\right| \leq \sum_{k=1}^{n}\left|z_{k}-w_{k}\right|,
$$

for $z_{k}, w_{k} \in[-1,1], k=1,2, \ldots, n$, (see for example Lemma 3 in [4]) and that $\left|\mathrm{e}^{u}-1-u\right| \leq u^{2}$ for $|u| \leq 1 / 2$.

Proof of Theorem 1. By the assumptions the immigration $\varepsilon_{n}$ has $\operatorname{Bernoulli}\left(m_{n, 1}\right)$ distribution, that is $H_{n}(x)=1+m_{n, 1}(x-1)$, in which case formula (1) reduces to

$$
F_{n}(x)=\prod_{j=1}^{n}\left[1+m_{j, 1}\left(\bar{G}_{j+1, n}(x)-1\right)\right]
$$

Let us define the generating function

$$
\tilde{F}_{n}(x)=\prod_{j=1}^{n} \mathrm{e}^{m_{j, 1}\left(\bar{G}_{j+1, n}(x)-1\right)} .
$$

Estimation (9) gives that if $m_{n, 1} \rightarrow 0$ then $\max _{1 \leq j \leq n} m_{j, 1}\left|\bar{G}_{j+1, n}(x)-1\right| \rightarrow 0$.

We want to show that for all $x \in(0,1)$

(a) $\tilde{F}_{n}(x)-F_{n}(x) \rightarrow 0$, as $n \rightarrow \infty$, and

(b) $\lim _{n \rightarrow \infty} \tilde{F}_{n}(x)=\mathrm{e}^{\lambda(x-1)}$.

Condition (a) is the easier to handle. Using (8), the inequality $\mid \mathrm{e}^{u}-1-$ $u \mid \leq u^{2}$ and the mean value theorem combined with the monotonicity of the 
derivative, we obtain

$$
\begin{aligned}
\left|\tilde{F}_{n}(x)-F_{n}(x)\right| & =\left|\prod_{j=1}^{n} \mathrm{e}^{m_{j, 1}\left(\bar{G}_{j+1, n}(x)-1\right)}-\prod_{j=1}^{n}\left[1+m_{j, 1}\left(\bar{G}_{j+1, n}(x)-1\right)\right]\right| \\
& \leq \sum_{j=1}^{n}\left|\mathrm{e}^{m_{j, 1}\left(\bar{G}_{j+1, n}(x)-1\right)}-\left[1+m_{j, 1}\left(\bar{G}_{j+1, n}(x)-1\right)\right]\right| \\
& \leq \sum_{j=1}^{n} m_{j, 1}^{2}\left|\bar{G}_{j+1, n}(x)-1\right|^{2} \\
& \leq \sum_{j=1}^{n} m_{j, 1}^{2}|x-1|^{2}\left(\bar{G}_{j+1, n}^{\prime}(1)\right)^{2} .
\end{aligned}
$$

Using (2), finally we have

$$
\left|\tilde{F}_{n}(x)-F_{n}(x)\right| \leq|x-1|^{2} \sum_{j=1}^{n} m_{j, 1}^{2} \rho_{[j, n]}^{2},
$$

and by Lemma 5 in [4] this goes to 0 under the assumptions of the theorem.

Now consider condition (b). This convergence is equivalent to

$$
\sum_{j=1}^{n} m_{j, 1}\left[\bar{G}_{j+1, n}(x)-1\right] \rightarrow \lambda(x-1) .
$$

Let $x \in(0,1)$. The convexity of the generating functions implies $G_{n}(x) \geq$ $1-\rho_{n}+\rho_{n} x=1+\rho_{n}(x-1)$. And so

$$
\bar{G}_{n-1, n}(x)=G_{n-1}\left(G_{n}(x)\right) \geq 1+\rho_{n-1}\left(G_{n}(x)-1\right) \geq 1+\rho_{n-1} \rho_{n}(x-1) .
$$

Induction gives that

$$
\bar{G}_{j+1, n}(x) \geq 1+\rho_{j+1} \rho_{j+2} \cdots \rho_{n}(x-1)=1+\rho_{[j, n]}(x-1),
$$

which implies the lower bound

$$
\sum_{j=1}^{n} m_{j, 1}\left[\bar{G}_{j+1, n}(x)-1\right] \geq \sum_{j=1}^{n} m_{j, 1} \rho_{[j, n]}(x-1) \rightarrow \lambda(x-1) .
$$


For the upper bound note that by the previous estimations $\bar{G}_{j+1, n}(x) \in$ $\left(1-\rho_{[j, n]}, 1\right)$ for any $x \in(0,1)$. Again by convexity, for $y \in\left(1-\rho_{[j, n]}, 1\right)$ we have

$$
\begin{aligned}
G_{j}(y) & =G_{j}\left(\frac{1-y}{\rho_{[j, n]}}\left(1-\rho_{[j, n]}\right)+1-\frac{1-y}{\rho_{[j, n]}}\right) \\
& \leq \frac{1-y}{\rho_{[j, n]}} G_{j}\left(1-\rho_{[j, n]}\right)+1-\frac{1-y}{\rho_{[j, n]}} \\
& =1+\vartheta_{j, n}(y-1),
\end{aligned}
$$

where

$$
\vartheta_{j, n}=\frac{1-G_{j}\left(1-\rho_{[j, n]}\right)}{\rho_{[j, n]}}=G_{j}^{\prime}\left(\xi_{j, n}\right),
$$

by the mean value theorem. Since $\rho_{[j, n]} \rightarrow 0$ thus $\xi_{j, n} \rightarrow 1$ as $n \rightarrow \infty$, $\vartheta_{j, n} \uparrow \rho_{j}$. So we get

$$
\bar{G}_{j, n}(x)=G_{j}\left(\bar{G}_{j+1, n}(x)\right) \leq 1+\vartheta_{j, n}\left(\bar{G}_{j+1, n}(x)-1\right),
$$

and so induction gives

$$
\bar{G}_{j+1, n}(x) \leq 1+\vartheta_{j+1, n} \vartheta_{j+2, n} \cdots \vartheta_{n, n}(x-1)=: 1+\vartheta_{[j, n]}(x-1) .
$$

Summarizing we have

$$
\sum_{j=1}^{n} m_{j, 1}\left[\bar{G}_{j+1, n}(x)-1\right] \leq \sum_{j=1}^{n} m_{j, 1} \vartheta_{[j, n]}(x-1) .
$$

Note that in the case of Bernoulli offspring distributions the upper and lower bounds are equal.

So we have to check that under what conditions

$$
\sum_{j=1}^{n} m_{j, 1} \vartheta_{[j, n]} \rightarrow \lambda
$$

For this, exactly the same way as in Lemma 5 in [4] we only need that the sequence $b_{n, j}:=\left(1-\rho_{j}\right) \vartheta_{[j, n]}$ form a Toeplitz matrix. The only nontrivial condition is

$$
\sum_{j=1}^{n} b_{n, j} \rightarrow 1
$$


We may write

$$
\begin{aligned}
\sum_{j=1}^{n} b_{n, j}=\left(1-\rho_{1}\right) \prod_{l=2}^{n} \vartheta_{l, n} & +\left(1-\rho_{2}\right) \prod_{l=3}^{n} \vartheta_{l, n}+\cdots \\
& +\left(1-\rho_{n-1}\right) \vartheta_{n, n}+\left(1-\rho_{n}\right) \\
=1-\left[\left(\rho_{n}-\vartheta_{n, n}\right)\right. & +\left(\rho_{n-1}-\vartheta_{n-1, n}\right) \vartheta_{n, n}+\cdots \\
& \left.+\left(\rho_{2}-\vartheta_{2, n}\right) \prod_{l=3}^{n} \vartheta_{l, n}+\rho_{1} \prod_{l=2}^{n} \vartheta_{l, n}\right] .
\end{aligned}
$$

Note that in the bracket every term is nonnegative, since $\vartheta_{j, n}=G_{j}^{\prime}\left(\xi_{j, n}\right) \leq$ $G_{j}^{\prime}(1)=\rho_{j}$, because $G_{j}^{\prime}$ is monotone increasing since $G_{j}$ is convex. So we need that

$$
\left(\rho_{n}-\vartheta_{n, n}\right)+\left(\rho_{n-1}-\vartheta_{n-1, n}\right) \vartheta_{n, n}+\cdots+\left(\rho_{2}-\vartheta_{2, n}\right) \prod_{l=3}^{n} \vartheta_{l, n}+\rho_{1} \prod_{l=2}^{n} \vartheta_{l, n} \rightarrow 0
$$

By definition we have $\vartheta_{j, n}=G_{j}^{\prime}\left(\xi_{j, n}\right)$, where $\xi_{j, n} \in\left(1-\rho_{[j, n]}, 1\right)$, and since $\rho_{j}=G_{j}^{\prime}(1)$ the mean value theorem again gives

$$
\rho_{j}-\vartheta_{j, n}=G_{j}^{\prime}(1)-G_{j}^{\prime}\left(\xi_{j, n}\right)=\left(1-\xi_{j, n}\right) G_{j}^{\prime \prime}\left(\xi_{j, n}^{\prime}\right) \leq \rho_{[j, n]} G_{j}^{\prime \prime}(1),
$$

where $\xi_{j, n}^{\prime} \in\left(\xi_{j, n}, 1\right)$. Therefore we obtain

$$
\sum_{j=1}^{n}\left(\rho_{j}-\vartheta_{j, n}\right) \vartheta_{[j, n]} \leq \sum_{j=1}^{n} \rho_{[j, n]} G_{j}^{\prime \prime}(1) \vartheta_{[j, n]} \leq \sum_{j=1}^{n} \frac{G_{j}^{\prime \prime}(1)}{1-\rho_{j}}\left(1-\rho_{j}\right) \rho_{[j, n]}^{2},
$$

which, according to Lemma 5 [4], goes to 0 , if $\frac{G_{n}^{\prime \prime}(1)}{1-\rho_{n}} \rightarrow 0$. The proof is ready.

Proof of Theorem 4. By formula (1) for the generating function we have to show that for all $x \in(0,1)$

$$
F_{n}(x)=\prod_{j=1}^{n} H_{j}\left(\bar{G}_{j+1, n}(x)\right) \rightarrow \exp \left\{\sum_{l=1}^{\infty} \frac{(x-1)^{l}}{l !} \lambda_{l}\right\} .
$$

Let us define the function

$$
\tilde{F}_{n}(x)=\prod_{j=1}^{n} \mathrm{e}^{H_{j}\left(\bar{G}_{j+1, n}(x)\right)-1}
$$


Using the estimation (9) and that $0 \leq 1-H_{j}(x) \leq m_{j, 1}(1-x)$, we have

$$
\begin{aligned}
\left|F_{n}(x)-\tilde{F}_{n}(x)\right| & \leq \sum_{j=1}^{n}\left|\mathrm{e}^{H_{j}\left(\bar{G}_{j+1, n}(x)\right)-1}-H_{j}\left(\bar{G}_{j+1, n}(x)\right)\right| \\
& \leq \sum_{j=1}^{n}\left(H_{j}\left(\bar{G}_{j+1, n}(x)\right)-1\right)^{2} \\
& \leq \sum_{j=1}^{n} m_{j, 1}^{2} \rho_{[j, n]}^{2} \rightarrow 0,
\end{aligned}
$$

since $m_{j, 1} /\left(1-\rho_{j}\right) \rightarrow \lambda_{1}$ implies $m_{j, 1}^{2} /\left(1-\rho_{j}\right) \rightarrow 0$. So we obtain the convergence $F_{n}(x)-\tilde{F}_{n}(x) \rightarrow 0$, for all $x \in(0,1)$. Therefore what we have to show is that

$$
\sum_{j=1}^{n}\left[H_{j}\left(\bar{G}_{j+1, n}(x)\right)-1\right] \rightarrow \sum_{l=1}^{\infty} \frac{(x-1)^{l}}{l !} \lambda_{l} .
$$

Let us fix an $\varepsilon>0$ and an $x \in(0,1)$. There is an $l_{0}$ such that

$$
\left|\sum_{l=l_{0}+1}^{\infty} \frac{(x-1)^{l}}{l !} \lambda_{l}\right| \leq \varepsilon \quad \text { and } \quad \frac{(1-x)^{l_{0}+1}}{\left(l_{0}+1\right) !} \lambda_{l_{0}+1} \leq \varepsilon .
$$

By Lemma 6 in [4] we have

$$
H_{j}(x)=\sum_{l=0}^{l_{0}} \frac{m_{j, l}}{l !}(x-1)^{l}+R_{j, l_{0}+1}(x),
$$


where $\left|R_{j, l_{0}+1}(x)\right| \leq(1-x)^{l_{0}+1} m_{j, l_{0}+1} /\left(l_{0}+1\right)$ !. Thus

$$
\begin{aligned}
& \sum_{j=1}^{n}\left[H_{j}\left(\bar{G}_{j+1, n}(x)\right)-1\right] \\
= & \sum_{j=1}^{n}\left\{\sum_{l=1}^{l_{0}} \frac{m_{j, l}}{l !}\left(\bar{G}_{j+1, n}(x)-1\right)^{l}+R_{j, l_{0}+1}\left(\bar{G}_{j+1, n}(x)\right)\right\} \\
= & \sum_{l=1}^{l_{0}} \frac{(x-1)^{l}}{l !} \sum_{j=1}^{n} m_{j, l} \rho_{[j, n]}^{l}+\sum_{j=1}^{n} R_{j, l_{0}+1}\left(\bar{G}_{j+1, n}(x)\right) \\
& +\sum_{l=1}^{l_{0}} \sum_{j=1}^{n} \frac{m_{j, l}}{l !}\left[\left(\bar{G}_{j+1, n}(x)-1\right)^{l}-\rho_{[j, n]}^{l}(x-1)^{l}\right] \\
= & \sum_{l=1}^{l_{0}} \frac{(x-1)^{l}}{l !} \sum_{j=1}^{n} m_{j, l} \rho_{[j, n]}^{l}+I_{1}+I_{2} .
\end{aligned}
$$

Using (9) again

$$
\begin{aligned}
\left|I_{1}\right| & \leq \sum_{j=1}^{n} \frac{m_{j, l_{0}+1}}{\left(l_{0}+1\right) !}\left|\bar{G}_{j+1, n}(x)-1\right|^{l_{0}+1} \\
& \leq \sum_{j=1}^{n} \frac{m_{j, l_{0}+1}}{\left(l_{0}+1\right) !} \rho_{[j, n]}^{l_{0}+1}(1-x)^{l_{0}+1} \rightarrow \frac{(1-x)^{l_{0}+1}}{\left(l_{0}+1\right) !} \lambda_{l_{0}+1}
\end{aligned}
$$

by Lemma 5 in [4], thus (11), the choice of $l_{0}$, shows that $\left|I_{1}\right| \leq 2 \varepsilon$ for $n$ large enough. In order to estimate $I_{2}$ we use the inequalities (9) and (10) we have

$$
\begin{aligned}
& \left|\left(\bar{G}_{j+1, n}(x)-1\right)^{l}-\rho_{[j, n]}^{l}(x-1)^{l}\right| \\
& \leq\left|\left(\bar{G}_{j+1, n}(x)-1\right)-\rho_{[j, n]}(x-1)\right| l \rho_{[j, n]}^{l-1}(1-x)^{l-1} \\
& \leq l(1-x)^{l}\left(\rho_{[j, n]}-\vartheta_{[j, n]}\right) \rho_{[j, n]}^{l-1},
\end{aligned}
$$

therefore

$$
\left|I_{2}\right| \leq \sum_{l=1}^{l_{0}} \frac{(1-x)^{l}}{(l-1) !} \sum_{j=1}^{n} m_{j, l}\left(\rho_{[j, n]}-\vartheta_{[j, n]}\right) \rho_{[j, n]}^{l-1},
$$

which goes to 0, due to Lemma 5 in [4] and to our assumptions. 
Finally for the difference

$$
\begin{aligned}
& \left|\sum_{j=1}^{n}\left[H_{j}\left(\bar{G}_{j+1, n}(x)\right)-1\right]-\sum_{l=1}^{\infty} \frac{(x-1)^{l}}{l !} \lambda_{l}\right| \\
& \leq\left|\sum_{l=1}^{l_{0}} \frac{(x-1)^{l}}{l !}\left(\sum_{j=1}^{n} m_{j, l} \rho_{[j, n]}^{l}-\lambda_{l}\right)\right|+\left|\sum_{l=l_{0}+1}^{\infty} \frac{(x-1)^{l}}{l !} \lambda_{l}\right|+\left|I_{1}\right|+\left|I_{2}\right|,
\end{aligned}
$$

where the first and fourth term converge to 0 , while the sum of second and the third is less than $3 \varepsilon$. Since $\varepsilon$ was arbitrary the proof is ready.

Proof of Theorem 5. We separate three cases. First we assume that the offspring generating functions are second degree polynomials, that is each particle has at most two offsprings. Then we extend the proof when for the higher derivatives only assumption (iii) holds. In this two cases we assume Bernoulli immigration. In the third case we extend the result for general immigration. Step 1 shows the main idea without the technical difficulty to handle the higher derivatives.

Step 1. Let us assume that

$$
\operatorname{deg} G_{n}=2 \text { for all } n \in \mathbb{N} .
$$

First we obtain a recursion like (7). What we need is a general form of a derivative of a composite function. Let

$$
h_{k}(x)=\frac{\mathrm{d}^{k}}{\mathrm{~d} x^{k}} f(g(x)) .
$$

According to Lemma 5.6 in [5] the general formula for the derivatives of a composite function is

$$
\frac{\mathrm{d}^{k}}{\mathrm{~d} x^{k}} f(g(x))=k ! \sum_{s=1}^{k} f^{(s)}(g(x)) \sum_{\left(\nu_{1}, \ldots, \nu_{k}\right)} \prod_{m=1}^{k} \frac{1}{\nu_{m} !}\left(\frac{1}{m !} g^{(m)}(x)\right)^{\nu_{m}},
$$

where the summation is carried out over all nonnegative integer solutions of the equation system:

$$
\begin{aligned}
& \nu_{1}+2 \nu_{2}+\cdots+k \nu_{k}=k \\
& \nu_{1}+\nu_{2}+\cdots+\nu_{k}=s .
\end{aligned}
$$


Clearly the coefficient of $f^{\prime}(g)$ in $h_{k}$ is $g^{(k)}$, and induction argument shows that

coefficient of $f^{\prime \prime}(g)$ in $h_{k}= \begin{cases}\sum_{i=1}^{\frac{k-1}{2}}\left(\begin{array}{c}k \\ i\end{array}\right) g^{(i)} g^{(k-i)}, & k \text { odd }, \\ \sum_{i=1}^{\frac{k}{2}-1}\left(\begin{array}{c}k \\ i\end{array}\right) g^{(i)} g^{(k-i)}+\frac{1}{2}\left(\begin{array}{c}k \\ k / 2\end{array}\right)\left(g^{\left(\frac{k}{2}\right)}\right)^{2}, & k \text { even. }\end{cases}$

For simplicity introduce

$$
a_{k, i}= \begin{cases}\left(\begin{array}{c}
k \\
i
\end{array}\right), & \text { if } i<\frac{k}{2} \\
\frac{1}{2}\left(\begin{array}{c}
k \\
k / 2
\end{array}\right), & \text { if } i=\frac{k}{2} .\end{cases}
$$

Apply this result to $\bar{G}_{j, n}(x)=G_{j}\left(\bar{G}_{j+1, n}(x)\right)$ for $j \leq n$, and note that the third and higher derivatives vanishes. Substituting $x=1$ we have

$$
\bar{G}_{j, n}^{(k)}(1)=\rho_{j} \bar{G}_{j+1, n}^{(k)}(1)+G_{j}^{\prime \prime}(1) \sum_{i=1}^{k / 2} a_{k, i} \bar{G}_{j+1, n}^{(i)}(1) \bar{G}_{j+1, n}^{(k-i)}(1) .
$$

From this, easy induction argument gives the following:

$$
\bar{G}_{j+1, n}^{(k)}(1)=\sum_{i=1}^{k / 2} a_{k, i} \sum_{l=j+1}^{n} \rho_{[j, l-1]} G_{l}^{\prime \prime}(1) \bar{G}_{l+1, n}^{(i)}(1) \bar{G}_{l+1, n}^{(k-i)}(1) .
$$

Until now everything hold in general. Now we use our assumptions to prove

$$
\bar{G}_{j+1, n}^{(k)}(1)=\left(\frac{k !}{2^{k-1}} \nu^{k-1}+o(1)\right) \rho_{[j, n]}\left(1-\rho_{[j, n]}\right)^{k-1}+\rho_{[j, n]} O(1),
$$

where $o(1) \rightarrow 0$ as $j, n \rightarrow \infty$. The proof goes by induction, uses the recursion (15) and the identity

$$
\frac{(k+1) !}{2^{k}}=\frac{1}{k} \sum_{i=1}^{(k+1) / 2} a_{k+1, i} \frac{i !}{2^{i-1}} \frac{(k+1-i) !}{2^{k-i}} .
$$

Formula (16) is true for $k=1,2$, and let us assume that it is true until some 
$k \geq 2$. Then using the induction hypothesis

$$
\begin{aligned}
\bar{G}_{j+1, n}^{(k+1)}(1)= & \sum_{i=1}^{\frac{k+1}{2}} a_{k+1, i} \sum_{l=j+1}^{n} \rho_{[j, l-1]} G_{l}^{\prime \prime}(1) \bar{G}_{l+1, n}^{(i)}(1) \bar{G}_{l+1, n}^{(k+1-i)}(1) \\
= & \sum_{i=1}^{\frac{k+1}{2}} a_{k+1, i} \sum_{l=j+1}^{n} \rho_{[j, l-1]} G_{l}^{\prime \prime}(1) \\
& \times\left[\left(\frac{i !}{2^{i-1}} \nu^{i-1}+o(1)\right) \rho_{[l, n]}\left(1-\rho_{[l, n]}\right)^{i-1}+\rho_{[l, n]} O(1)\right] \\
& \times\left[\left(\frac{(k-i+1) !}{2^{k-i}} \nu^{k-i}+o(1)\right) \rho_{[l, n]}\left(1-\rho_{[l, n]}\right)^{k-i}+\rho_{[l, n]} O(1)\right] \\
= & \sum_{i=1}^{\frac{k+1}{2}} a_{k+1, i} \rho_{[j, n]} \sum_{l=j+1}^{n} \frac{G_{l}^{\prime \prime}(1)}{\rho_{l}\left(1-\rho_{l}\right)}\left(1-\rho_{l}\right) \rho_{[l, n]} \\
& \times\left[\left(\frac{(k+i-1) ! i !}{2^{k-1}} \nu^{k-1}+o(1)\right)\left(1-\rho_{[l, n]}\right)^{k-1}+o(1)\right] \\
= & \left(\frac{(k+1) !}{2^{k}} \nu^{k}+o(1)\right) \rho_{[j, n]}\left(1-\rho_{[j, n]}\right)^{k}+\rho_{[j, n]} o(1),
\end{aligned}
$$

where we used the estimation

$$
\sum_{l=j+1}^{n}\left(1-\rho_{l}\right) \rho_{[l, n]}\left(1-\rho_{[l, n]}\right)^{k}=\frac{\left(1-\rho_{[j, n]}\right)^{k+1}}{k+1}+O\left(\max _{j \leq l \leq n}\left(1-\rho_{l}\right) \rho_{[l, n]}\right) .
$$

Note that the left side is a Riemann approximation of the integral $\int(1-y)^{k} \mathrm{~d} y$, corresponding to the partition $\left\{\rho_{[l, n]}\right\}_{l=j+1}^{n}$. So (16) is proved.

From (16) using also assumption (iv) in the theorem we have

$$
\begin{aligned}
\sum_{j=1}^{n} m_{j, 1} \bar{G}_{j+1, n}^{(k)}(1)= & \sum_{j=1}^{n} \frac{m_{j, 1}}{1-\rho_{j}}\left(\frac{k !}{2^{k-1}} \nu^{k-1}+o(1)\right) \rho_{[j, n]}\left(1-\rho_{j}\right)\left(1-\rho_{[j, n]}\right)^{k-1} \\
& +\sum_{j=1}^{n} \frac{m_{j, 1}}{1-\rho_{j}}\left(1-\rho_{j}\right) \rho_{[j, n]} O(1) \\
\rightarrow & \frac{(k-1) !}{2^{k-1}} \lambda \nu^{k-1} \\
= & (k-1) ! \lambda\left(\frac{\nu}{2}\right)^{k-1} .
\end{aligned}
$$


So for the sum (5) we have

$$
\begin{aligned}
\sum_{j=1}^{n} m_{j, 1}\left(\bar{G}_{j+1, n}(x)-1\right) & =\sum_{j=1}^{n} m_{j, 1} \sum_{k=1}^{\infty} \frac{\bar{G}_{j+1, n}^{(k)}(1)}{k !}(x-1)^{k} \\
& =\sum_{k=1}^{\infty} \frac{(x-1)^{k}}{k !} \sum_{j=1}^{n} m_{j, 1} \bar{G}_{j+1, n}^{(k)}(1) \\
& \rightarrow \sum_{k=1}^{\infty} \frac{(x-1)^{k}}{k !}(k-1) ! \lambda\left(\frac{\nu}{2}\right)^{k-1} \\
& =-\frac{2 \lambda}{\nu} \log \left(1-(x-1) \frac{\nu}{2}\right),
\end{aligned}
$$

and Lemma 6 in [4] makes the calculation rigorous. Note that since $\bar{G}_{k, n}(x)$ is a polynomial the infinite sum above is in fact finite.

We obtained that the limit generating function is

$$
\exp \left\{-\frac{2 \lambda}{\nu} \log \left(1-(x-1) \frac{\nu}{2}\right)\right\}=\left(1-(x-1) \frac{\nu}{2}\right)^{-\frac{2 \lambda}{\nu}}=\left(\frac{\frac{2}{2+\nu}}{1-\frac{\nu}{2+\nu} x}\right)^{\frac{2 \lambda}{\nu}}
$$

which is the generating function of a negative binomial distribution with parameter $r=2 \lambda / \nu$ and $p=\nu /(2+\nu)$, as we stated.

Step 2. Let us weaken the condition (12) on the generating functions.

From formula (13) induction argument shows that

$$
\bar{G}_{j+1, n}^{(k)}(1)=k ! \sum_{s=2}^{k} \sum_{l=j+1}^{n} \rho_{[j, l-1]} G_{l}^{(s)}(1) \sum_{\left(\nu_{1}, \ldots, \nu_{k}\right)} \prod_{k=1}^{m} \frac{1}{\nu_{m} !}\left(\frac{\bar{G}_{l+1, n}^{(m)}(1)}{m !}\right)^{\nu_{m}} .
$$

We claim that under assumption (iii) of the theorem (16) holds. By Step 1 , this is true for $k=1,2$. Assume that the statement is true until some $k \geq 2$. The previous case shows that we get the asymptotic from the term corresponding to $s=2$, and we show that the terms corresponding to $s \geq 3$ are $o\left(\rho_{[j, n]}\right)$. Using the induction hypothesis for the term corresponding to 
$s \geq 3$ above we have

$$
\begin{aligned}
& \sum_{l=j+1}^{n} \rho_{[j, l-1]} G_{l}^{(s)}(1) \sum_{\left(\nu_{1}, \ldots, \nu_{k}\right)} \prod_{k=1}^{n} \frac{1}{\nu_{m} !}\left(\frac{\bar{G}_{l+1, n}^{(m)}(1)}{m !}\right)^{\nu_{m}} \\
& \leq \sum_{l=j+1}^{n} \rho_{[j, l-1]} G_{l}^{(s)}(1) \sum_{\left(\nu_{1}, \ldots, \nu_{k}\right)} \prod_{k=1}^{m} \operatorname{const} \rho_{[l, n]}^{\nu_{m}} \\
& =\operatorname{const} \rho_{[j, n]} \sum_{l=j+1}^{n} \frac{G_{l}^{(s)}(1)}{\rho_{l}\left(1-\rho_{l}\right)}\left(1-\rho_{l}\right) \rho_{[l, n]}^{s-1} \\
& =\rho_{[j, n]} o(1) .
\end{aligned}
$$

We proved (16), and the statement follows as in the previous case.

Step 3. Finally, in case of general immigration distribution, according to the already proved part what we have to show is

$$
\begin{aligned}
\left|\prod_{j=1}^{n} H_{j}\left(\bar{G}_{j+1, n}(x)\right)-\prod_{j=1}^{n}\left[1+m_{j, 1}\left(\bar{G}_{j+1, n}(x)-1\right)\right]\right| \\
\quad \leq \sum_{j=1}^{n}\left|H_{j}\left(\bar{G}_{j+1, n}(x)\right)-\left[1+m_{j, 1}\left(\bar{G}_{j+1, n}(x)-1\right)\right]\right| \\
\leq \sum_{j=1}^{n} \frac{m_{j, 2}}{2}\left(\bar{G}_{j+1, n}(x)-1\right)^{2} \\
\leq \frac{(x-1)^{2}}{2} \sum_{j=1}^{n} m_{j, 2} \rho_{[j, n]}^{2} \rightarrow 0,
\end{aligned}
$$

where we used Lemma 5 and 6 in [4], and the assumption $m_{n, 2} /\left(1-\rho_{n}\right) \rightarrow 0$. The proof is complete.

Proof of Theorem 6. The generating function of the $n^{\text {th }}$ generation is

$$
F_{n}(x)=\prod_{j=1}^{n} H_{j}\left(\bar{G}_{j+1, n}(x)\right) .
$$

First we show the existence of $\bar{G}_{j, \infty}(x)$. Let us fix $j$, and investigate $\bar{G}_{j, n}(x)$ as $n \rightarrow \infty$. Using the definition and the monotonicity of $\bar{G}_{j, n}$ and that $G_{n}(x) \geq x$ for all $n$, we have

$$
\bar{G}_{j, n+1}(x)=\bar{G}_{j, n}\left(G_{n+1}(x)\right) \geq \bar{G}_{j, n}(x) .
$$


Since $\bar{G}_{j, n}(x) \leq 1$, the limit

$$
\bar{G}_{j, \infty}(x)=\lim _{n \rightarrow \infty} \bar{G}_{j, n}(x)
$$

exists. Moreover $\bar{G}_{j+1, n}(x) \geq 1-\rho_{[j, n]}(1-x)$ implies

$$
\bar{G}_{j+1, \infty}(x) \geq 1-\frac{\rho(1-x)}{\rho_{[1, j]}} .
$$

Therefore we can define the function $g$ in the theorem.

Using the inequality above, the convergence $m_{j, 1} \rightarrow 0$, the estimation $H_{j}(x) \geq 1-m_{j, 1}(1-x)$ and that for $x$ small enough $1-x \geq \mathrm{e}^{-(1+\varepsilon) x}$ we obtain

$$
\begin{aligned}
H_{j}\left(\bar{G}_{j+1, \infty}(x)\right) & \geq 1-m_{j, 1}\left(1-\bar{G}_{j+1, \infty}(x)\right) \geq 1-m_{j, 1} \frac{\rho(1-x)}{\rho_{[1, j]}} \\
& \geq \exp \left\{-(1+\varepsilon) m_{j, 1} \frac{\rho(1-x)}{\rho_{[1, j]}}\right\} .
\end{aligned}
$$

This easily implies that the function $g$ is continuous at $1, g(1)=1$.

Next we show that $F_{n}(x) \rightarrow g(x)$, for all $x \in[0,1]$. Introduce the notation

$$
g_{n}(x)=\prod_{j=1}^{n} H_{j}\left(\bar{G}_{j+1, \infty}(x)\right) .
$$

Clearly $g_{n}(x) \rightarrow g(x)$, so we only have to show the convergence $g_{n}(x)-$ $F_{n}(x) \rightarrow 0$. We have

$$
\begin{aligned}
\left|g_{n}(x)-F_{n}(x)\right| & \leq \sum_{j=1}^{n}\left|H_{j}\left(\bar{G}_{j+1, \infty}(x)\right)-H_{j}\left(\bar{G}_{j+1, n}(x)\right)\right| \\
& \leq \sum_{j=1}^{n} m_{j, 1}\left|\bar{G}_{j+1, n}(x)-\bar{G}_{j+1, \infty}(x)\right| \\
& \leq \sum_{j=1}^{n_{0}} m_{j, 1}\left|\bar{G}_{j+1, n}(x)-\bar{G}_{j+1, \infty}(x)\right|+2 \sum_{j=n_{0}+1}^{\infty} m_{j, 1},
\end{aligned}
$$

where the first term goes to 0 for every fixed $n_{0}$, while the second one can be arbitrary small by choosing $n_{0}$ large enough.

\section{Acknowledgement}

I am grateful to Vincent Bansaye, who suggested to take a look at the linear fractional case, which eventually lead to the final form of Theorem 5 . 


\section{References}

[1] K. B. Athreya and P. E. Ney, Branching Processes. Springer, New York, 1972.

[2] W. Feller, An Introduction to Probability Theory and its Applications. Vol. I. Third edition John Wiley \& Sons, Inc., New York-London-Sydney 1968.

[3] J. H. Foster and J. A. Williamson, Limit Theorems for Galton-Watson Process with Time-Dependent Immigration. Z. Wahrsch. Verw. Gebiete 20, (1971), 227-235.

[4] L. Györfi, M. Ispány, G. Pap and K. Varga, Poisson limit of an inhomogeneous nearly critical INAR(1) model. Acta Sci. Math. (Szeged) 73(3-4), (2007), 789-815.

[5] V. V. Petrov, Limit Theorems of Probability Theory. Oxford Studies in Probability 4, Clarendon Press, Oxford, 1995. 